\title{
Proteinase 3 activity in sputum from subjects with alpha-1-antitrypsin deficiency and COPD
}

\author{
Nicola J. Sinden* and Robert A. Stockley\#
}

ABSTRACT: Chronic obstructive pulmonary disease (COPD) is associated with tissue damage believed to result from an imbalance between serine proteinases and their inhibitors. Although the role of neutrophil elastase (NE) has been studied, it is likely that other proteinases play a role. The importance of proteinase 3 (PR3) has not been established, as specific substrates have only recently been available.

We studied clinically stable subjects with either alpha-1-antitrypsin (A1AT) deficiency or usual COPD with chronic bronchitis. Sol phase sputum was analysed for PR3 activity and concentration, NE activity and concentration, concentrations of airway inhibitors (A1AT, secretory leukoproteinase inhibitor and elafin) and markers of neutrophilic inflammation. 12 patients were also studied during exacerbations.

PR3 activity was present in most sputum samples and greater than NE activity (which was largely undetectable) in both subject groups (A1AT deficiency median PR3 $128 \mathrm{nM}$, interquartile range (IQR) 33-558 nM; NE 0 nM, IQR 0-0 nM; p=0.0043; COPD PR3 22 nM, IQR 0-103 nM; NE $0 \mathrm{nM}$, IQR 0-0 nM; $\mathrm{p}=0.015)$. PR3 activity was greater during exacerbations than in the stable state $(p=0.037)$ and correlated with markers of neutrophilic inflammation.

The regular identification of PR3 activity in sputum from stable subjects with A1AT deficiency or usual COPD suggests it may play a greater role in the pathophysiology than previously thought.

\section{KEYWORDS: Chronic obstructive pulmonary disease, inflammatory mediators, proteinases}

hronic obstructive pulmonary disease (COPD) is a major cause of morbidity and mortality worldwide [1]. Apart from smoking cessation and use of long term oxygen therapy in hypoxic patients, current therapies only provide symptomatic benefit, and there is little evidence that they alter disease progression or mortality. Novel therapies are therefore urgently needed to improve quality of life and prognosis.

The pathophysiology of the disease remains unclear although inflammation and tissue damage are thought to be central to the development of COPD. In particular, an imbalance between neutrophil serine proteinases (NSPs) and their inhibitors has been thought to play a key role [2]. This belief is based on the association of alpha-1antitrypsin (A1AT) deficiency (the major inhibitor of these proteinases) with early onset COPD [3], and the fact that neutrophil elastase (NE) and proteinase 3 (PR3) are able to degrade many critical components of the extracellular matrix, including elastin, type IV collagen, fibronectin and laminin $[4,5]$. In addition, these proteinases also produce emphysematous lesions when administered by intratracheal instillation in animal models $[6,7]$ and can produce other features of COPD including mucus hypersecretion [8]. The activities of the NSPs in the lungs are usually controlled by endogenous inhibitors, such as A1AT, which is mainly derived from the serum and inhibits NE and PR3, secretory leukoproteinase inhibitor (SLPI), which is mainly produced locally and inhibits NE but not PR3, and elafin and its precursor trappin-2 (also produced locally), which inhibit NE and PR3 [9].

The role of NE in the pathogenesis of COPD and, particularly, emphysema has been studied extensively [10]. However, selective inhibitors of NE have not been fully effective in controlling neutrophil-mediated damage in the airways [11], supporting the likelihood that other proteinases play a role. Further, KORKMAZ et al. [12] have suggested that A1AT preferentially inhibits NE, and that PR3 is only inhibited after NE is completely neutralised, suggesting that PR3 may play a more important role when both are released.
AFFILIATIONS

${ }^{*}$ Centre for Translational

Inflammation Research, University of Birmingham Research Laboratories, Queen Elizabeth Hospital,

Birmingham, and

\#The ADAPT project, Lung Function and Sleep Dept, Queen Elizabeth Hospital, Birmingham, UK.

CORRESPONDENCE

R.A. Stockley

Lung Function and Sleep Dept, ADAPT Office (Office 4)

Queen Elizabeth Hospital

Birmingham

B15 2WB

UK

E-mail: rob.stockley@uhb.nhs.uk

Received:

June 062012

Accepted after revision:

Aug 112012

First published online:

Aug 302012 
To date there is little direct evidence of the role of PR3, and research has been hindered by a lack of availability of specific substrates and inhibitors.

PR3 is a multifunctional serine proteinase mainly located in the azurophilic granules and on the cell surface of neutrophils [13]. It is a $29 \mathrm{kDa}$ glycoprotein alternatively named myeloblastin, AGP7 and p29b [14, 15]. Both PR3 and NE are released simultaneously upon neutrophil activation and degranulation. PR3 is, however, the most abundant serine proteinase in the neutrophil with each cell being estimated to store approximately three times more PR3 than NE [16]. A pro-inflammatory role for PR3 has been suggested by its ability to activate tumour necrosis factor- $\alpha$ and interleukin (IL)-1 $\beta$ [17] and provoke apoptosis [18]. The amino acid sequence and crystal structure of PR3 is similar to that of NE [19], and until recently no substrate was available that could discriminate between the two proteinases [20]. However, highly sensitive Abz-peptidyl-EDDnp fluorescence resonance energy transfer (FRET) substrates are now available that can differentiate these human NSPs and are capable of measuring subnanomolar concentrations in biological fluids [21].

The current study was designed to investigate the presence and activity of PR3 compared to NE in sol phase sputum from subjects with A1AT deficiency and non-deficient COPD, together with the concentrations of their cognate inhibitors. The results provide insight into the contribution of PR3 to the proteolytic activity in the lungs, and hence its potential role with implications as a therapeutic target.

\section{METHODS}

\section{Subject selection}

28 clinically stable subjects (at least 8 weeks after any acute infection) with A1AT deficiency (PiZZ confirmed by genotyping) with a history of chronic bronchitis were identified from the UK national registry for A1AT deficiency, and spontaneously produced sputum was collected as described previously [22]. None of the A1AT deficient subjects were receiving augmentation therapy. Chronic bronchitis was a feature in $35 \%$ of subjects on the UK national registry.

22 patients with usual COPD (normal PiMM A1AT phenotype) were recruited from primary care at the start of an acute exacerbation. These subjects had a clinical diagnosis of COPD based on a history of chronic bronchitis and exertional dyspnoea, with or without supportive spirometry at presentation (but subsequently confirmed to meet the diagnostic criteria [23]). Sputum was collected at the start of an acute exacerbation (day 1) and then after resolution of the episode during convalescence (day 56). In total, 12 day 1 samples and 22 day 56 samples of sufficient volume were available for the study.

All subjects had full demographic data collected including smoking history, full pulmonary function tests and a high resolution computed tomography scan performed in the stable clinical state. The A1AT deficient subjects had their health status assessed using the St George's Respiratory Questionnaire (SGRQ).

The study was approved by the local research ethics committee and all subjects gave informed consent.

\section{Preparation of sputum samples}

Spontaneous sputum samples were collected (as free from saliva as possible after a mouthwash) and divided into two aliquots; one aliquot was used to obtain quantitative microbiological culture as described previously [24], and the second was ultracentrifuged $\left(50000 \times g\right.$ for $90 \mathrm{~min}$ at $4^{\circ} \mathrm{C}$ ) to obtain the sol phase which was stored at $-70^{\circ} \mathrm{C}$ until analysis.

\section{Measurement of PR3 and NE activities in sol phase sputum}

The enzymatic activities of PR3 and NE in the samples were evaluated by measuring the hydrolysis of specific substrates using pure proteinases as standards. Pure PR3 (Merck, Feltham, UK) and pure NE (Athens Research and Technology, Athens, GA, USA) were active site titrated against pure A1AT (Athens Research and Technology), which had been previously titrated against porcine pancreatic elastase (PPE; Sigma-Aldrich, Gillingham, UK) of known activity. The activity of PPE was determined using Lineweaver-Burk double-reciprocal plot analysis with $N$-succinyl-Ala-AlaAla-p-nitroanilide (SlaaapN; Sigma-Aldrich) as the substrate and published kinetic constants [25].

The PR3 activity assays were performed using the FRET substrate Abz-VAD-norV-ADRQ-EDDnp (Alta Biosciences, Birmingham. UK), which was prepared as described previously [21]. This substrate has a catalytic constant $\mathrm{Kcat} / \mathrm{Km}$ of $3400 \mathrm{mM}^{-1} \cdot \mathrm{s}^{-1}$ and shows no significant hydrolysis by NE [26]. Pure PR3 was diluted in buffer (50 mM HEPES, pH 7.4, $150 \mathrm{mM} \mathrm{NaCl}, 0.05 \%$ Igepal CA-630) to an active concentration of $10 \mathrm{nM}$ and serially diluted in the same buffer to $0.625 \mathrm{nM}$. Buffer alone was used as a blank for the assay. Sol phase sputum samples were diluted 1 in 60 and the standards and samples $(150 \mu \mathrm{L})$ were added to a black opaque polypropylene low binding plate (Sigma-Aldrich) in duplicate. A 1 in 60 dilution was initially chosen as most values fell within the range of the standard curve. Subsequently, $3 \mu \mathrm{L}$ of $1 \mathrm{mM}$ FRET substrate was added to each well and the fluorescence was measured (excitation $320 \mathrm{~nm}$, emission $420 \mathrm{~nm}$ ) at regular intervals for as long as the curve was linear using a Biotek Synergy 2 Multi-Mode Microplate Reader (BioTek, Winooski, VT, USA) at $37^{\circ} \mathrm{C}$. The activities of PR3 in the samples were obtained by interpolation from the standard curve. Any sample with a value above or below that of the standard curve was repeated at a suitable dilution. Using this FRET substrate, reliable measurements were obtained for PR3 in the range $0.1-10 \mathrm{nM}$ [21]. The intra-assay coefficient of variation (CV) was $3.98 \%$ and the inter-assay CV was $13.35 \%$. All results were corrected for the initial sample dilution.

The NE activity assays were performed using SlaaapN as the substrate, which has a catalytic constant $\mathrm{Kcat} / \mathrm{Km}$ of $465 \mathrm{M}^{-1} \cdot \mathrm{s}^{-1}$ and shows no significant hydrolysis with PR3 [27]. Pure NE was diluted in buffer to $1 \mu \mathrm{M}$ and then serially diluted to $15.6 \mathrm{nM}$. Buffer alone was used as a blank for the assay. Sol phase sputum samples were studied undiluted with appropriate controls. The standards, samples and control samples (30 $\mu \mathrm{L})$ were added to a 96-well plate in duplicate and the substrate SlaaapN $\left(150 \mu \mathrm{L}\right.$ of $\left.1 \mathrm{mg} \cdot \mathrm{mL}^{-1}\right)$ was added, except for the control wells where buffer was added instead of substrate. 
The optical density (OD) at $410 \mathrm{~nm}$ was read at regular intervals up to $60 \mathrm{~min}$ using a Biotek Synergy HT plate reader (BioTek) at $37^{\circ} \mathrm{C}$. The activities of $\mathrm{NE}$ in the samples were obtained by interpolation from the standard curve after subtraction of control values. The lower limit of detection for this substrate was $15 \mathrm{nM}$ and the intra- and inter-assay CVs were $3.48 \%$ and $4.76 \%$ respectively. Any samples with values below this level were re-assayed using the NE specific FRET substrate Abz-APEEIMRRQ-EDDnp, providing reliable measurements for NE in the range $0.1-10 \mathrm{nM}$ [21]. The intra-assay CV was $3.42 \%$ and the inter-assay CV was $4.76 \%$.

The enzymatic activities of both Pseudomonas aeruginosa culture supernatants and pure Pseudomonas elastase (Merck) were assessed using the above substrates to confirm no cross-reactivity with the assays for human proteinases.

\section{Measurement of PR3 and NE concentrations in sol phase sputum}

The concentrations of PR3 and NE in the samples were measured by ELISA using commercially available kits. The PR3 ELISA (Biorbyt, Cambridge, UK) only detects unbound PR3, whereas the NE ELISA (Cambridge Bioscience, Cambridge, UK) detects both free NE and NE bound to inhibitors. The lower limits of detection were $5 \mathrm{pM}$ for the PR3 ELISA and $14 \mathrm{pM}$ for the NE ELISA. The intra- and inter-assay CVs for these ELISAs were $3.59 \%$ and $9.19 \%$, respectively, for the PR3 ELISA and $5.07 \%$ and $13.76 \%$, respectively, for the NE ELISA.

\section{Measurement of inhibitors in sol phase sputum}

The concentration of A1AT was measured using a locally developed ELISA. In brief, 96-well plates (Nunc Maxisorp; Sigma-Aldrich) were coated overnight with $200 \mu \mathrm{L}$ of $2 \mu \mathrm{L} \cdot \mathrm{mL}^{-1}$ goat anti-human A1AT antibody (Binding Site, Birmingham, UK) in carbonate buffer, $\mathrm{pH}$ 9.6. After blocking with $1 \%$ bovine serum albumin, sol phase sputum samples (diluted 1:100 to 1:10,000) were added and a human serum protein calibrator (Dako, Ely, UK) containing $1.24 \mathrm{~g} \cdot \mathrm{L}^{-1}$ A1AT was used to develop a range of standards $\left(1.9-62 \mathrm{ng} \cdot \mathrm{mL}^{-1}\right)$ and the plates were incubated for $2 \mathrm{~h}$ at room temperature. Following this, the next antibody $\left(200 \mu \mathrm{L}\right.$ of $2.6 \mu \mathrm{L} \cdot \mathrm{mL}^{-1}$ goat anti-human A1AT peroxidase conjugate (Binding Site) in PBS, $0.1 \%(\mathrm{w} / \mathrm{v})$ albumin, and $0.05 \%(\mathrm{v} / \mathrm{v})$ Tween 20$)$ was added and the plates incubated for $2 \mathrm{~h}$ at room temperature. The substrate, tetramethylbenzidine solution (Sigma-Aldrich), was then added and the reaction was stopped with $0.1 \mathrm{M} \mathrm{H}_{2} \mathrm{SO}_{4}$ when a suitable colour change had developed. The OD was then read at $450 \mathrm{~nm}$ with a $570 \mathrm{~nm}$ wavelength correction and sample concentration was determined from the standards by interpolation. The intra- and inter- assay CVs were $4.66 \%$ and $4.35 \%$, respectively.

The concentrations of SLPI and elafin were measured using commercially available ELISA kits (R\&D Systems, Abingdon, UK) according to the manufacturer's instructions. The intraand inter-assay CVs were $6.37 \%$ and $10.76 \%$, respectively, for the SLPI ELISA and $7.67 \%$ and $4.46 \%$, respectively, for the elafin ELISA.

\section{Other measurements in sol phase sputum}

Markers of neutrophilic inflammation were measured when sufficient sample remained, including IL-8, leukotriene (LT)-B4 and myeloperoxidase (MPO). IL-8 and LTB4 were measured using commercially available ELISA kits (R\&D Systems). The intra- and inter-assay CVs were $5.97 \%$ and $7.22 \%$, respectively, for the IL-8 ELISA and $6.34 \%$ and $11.9 \%$, respectively, for the LTB4 ELISA. MPO activity was measured as described previously [28]. The intra- and inter- assay CVs for the MPO activity assays were $3.85 \%$ and $10.07 \%$, respectively.

\section{Statistical analysis}

Statistical analyses were performed using PASW statistics 18 for Windows. Normality was tested using the KolmogorovSmirnov test. PR3 activity, PR3 concentration, NE activity, NE concentration, IL-8, LTB4, MPO, A1AT, SLPI, forced expiratory volume in $1 \mathrm{~s}$ (FEV1) and SGRQ total score were not normally distributed, therefore non-parametric tests were used and data are presented as medians and interquartile ranges (IQRs). Mann-Whitney U-tests or Wilcoxon signed rank tests were used for comparisons of independent or related data, respectively. Correlations were assessed using Spearman's rank correlation coefficient. Normally distributed data are presented as mean \pm SEM and independent or paired $t$-tests were used for comparisons of data for independent or related measures, respectively. To compare variables between A1AT deficient and COPD subjects after adjusting for baseline differences, the data were log transformed and linear regression was used with group as one of the independent factors. The unstandardised residuals were tested for normality. For statistical purposes, enzyme activity below the lower limit of detection was taken as $0.1 \mathrm{nM}$. Results were deemed statistically significant if $\mathrm{p} \leqslant 0.05$.

\section{RESULTS}

\section{Baseline characteristics}

Baseline characteristics of the two subject groups are shown in table 1. The A1AT deficient subjects were younger, had a lower smoking history (pack-years), FEV1 (\% predicted), FEV1/forced vital capacity (FVC) ratio and carbon monoxide transfer coefficient $(\mathrm{KCO})$ (\% predicted) compared to the COPD subjects. Of the 28 A1AT deficient subjects, seven were never smokers, 19 were ex-smokers and two were current smokers. Of the 22 COPD subjects, 15 were ex-smokers and seven were current smokers. There were no significant differences in sex distribution or radiological evidence of emphysema between the two groups.

\section{PR3 and NE activities in sputum from clinically stable subjects}

Detectable PR3 activity was found in all sputum samples from the A1AT deficient subjects (median $128 \mathrm{nM}$, IQR 33-558 nM) and in $64 \%$ of samples from the COPD subjects (median $22 \mathrm{nM}$, IQR 0-103 nM). PR3 activity was greater in A1AT deficient subjects compared to those with usual COPD $(p=0.004)$ after adjusting for baseline differences in age, smoking history (packyears), FEV1 (\% pred) and KCO (\% pred). Unbound PR3 concentration measured by ELISA confirmed these differences and the values correlated strongly with PR3 activity 


\begin{tabular}{lccc}
\hline TABLE 1 & Baseline characteristics & & \\
& $\begin{array}{c}\text { A1AT } \\
\text { deficiency }\end{array}$ & COPD & p-value \\
& $55 \pm 1.9$ & $65 \pm 1.9$ & 0.002 \\
\hline Age years & $22(79)$ & $19(86)$ & NS \\
Number of males & $21 \pm 3.9$ & $54 \pm 10.0$ & 0.004 \\
Smoking history & & & \\
$\quad$ pack-years & $49 \pm 5.9$ & $72 \pm 5.8$ & 0.004 \\
FEV1 \% pred & $0.41 \pm 0.04$ & $0.49 \pm 0.04$ & 0.031 \\
FEV1/FVC ratio & $69 \pm 5.2$ & $101 \pm 6.1$ & 0.001 \\
Kco \% pred & $22(79)$ & $14(64)$ & NS \\
HRCT evidence & & & \\
\hline of emphysema & & & \\
\hline
\end{tabular}

Data are presented as mean \pm SEM or $n(\%)$. A1AT: alpha-1-antitrypsin; COPD: chronic obstructive pulmonary disease; FEV1: forced expiratory volume in $1 \mathrm{~s}$; FVC: forced vital capacity; KCO: carbon monoxide transfer coefficient; HRCT: high-resolution computed tomography; NS: nonsignificant

(Spearman's rho 0.879, $\mathrm{p}<0.001$ ) indicating that most of the unbound PR3 was enzymatically active.

Detectable NE activity was found in $21 \%$ of A1AT deficient sputum samples (median $0 \mathrm{nM}$, IQR $0-0 \mathrm{nM}$ ) and $5 \%$ of usual COPD samples (median $0 \mathrm{nM}$, IQR $0-0 \mathrm{nM}$ ). The concentration of NE measured by ELISA (free and bound to inhibitors) was not significantly different $(p=0.296)$ between subjects with A1AT deficiency (median $330 \mathrm{nM}$, IQR 140-813 nM) and usual COPD (median $214 \mathrm{nM}$, IQR $84-564 \mathrm{nM}$ ). In the A1AT deficient group, three subjects had higher NE activity than PR3 activity. These three subjects were colonised with $P$. aeruginosa whilst none of the subjects with usual COPD were. Neither $P$. aeruginosa culture supernatants nor purified Pseudomonas elastase were able to hydrolyse the substrates used for the NE assays (data not shown), suggesting that the NE activity was specific. Of note, Pseudomonas elastase activity is not inhibited by A1AT or the other airway serine proteinase inhibitors [29].

The activity results are summarised in figure 1. PR3 activity was greater than NE activity in subjects with A1AT deficiency $(p=0.004)$ and those with usual COPD $(p=0.015)$.

\section{NSP inhibitory proteins in sputum}

As expected, the concentration of A1AT was higher in sputum from subjects with non-deficient COPD compared to those with A1AT deficiency (COPD median $405 \mathrm{nM}$, IQR 234$744 \mathrm{nM}$; A1AT deficiency median $51 \mathrm{nM}$, IQR 19-83 nM; $\mathrm{p}<0.001$ ). In addition, usual COPD subjects also had higher concentrations of SLPI (COPD median $2.8 \mu \mathrm{M}$, IQR 1.7-4.4 $\mu \mathrm{M}$; A1AT deficiency median $1.3 \mu \mathrm{M}$, IQR $0.8-2.7 \mu \mathrm{M}$; $\mathrm{p}=0.002$ ) and elafin (mean \pm SEM $316 \pm 37 \mathrm{pM}$ versus $200 \pm 39 \mathrm{pM}$; $\mathrm{p}=0.039$ ). SLPI was quantitatively the predominant NSP inhibitor in sputum from both subject groups. The contribution to the total airway inhibitory capacity of elafin was negligible, being found in subnanomolar concentrations in all samples. As shown in figure 2, airway inhibitors of PR3 were quantitatively less than those of NE.

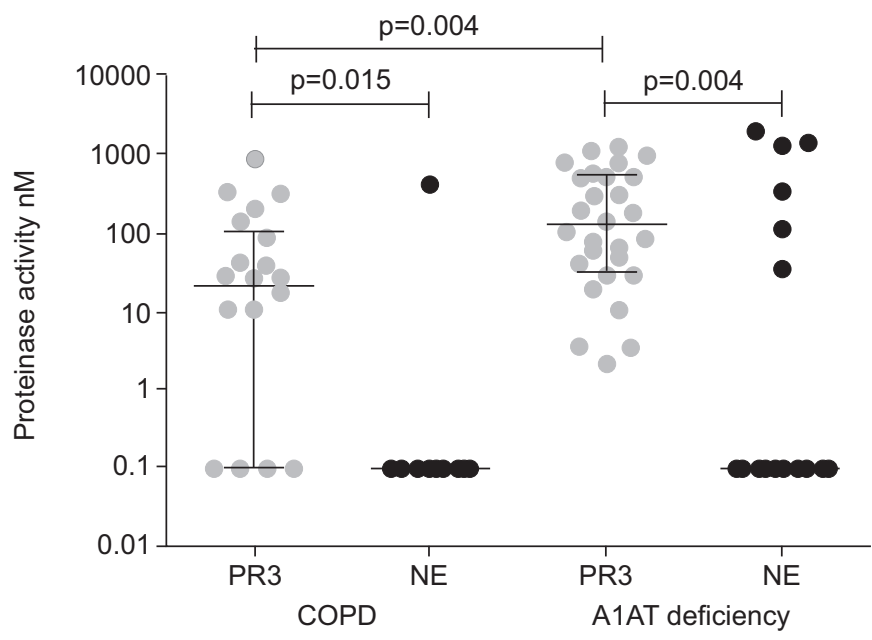

FIGURE 1. Proteinase 3 (PR3) and neutrophil elastase (NE) activities in sol phase sputum spontaneously produced by clinically stable subjects with alpha-1antitrypsin (A1AT) deficiency $(n=28)$ and chronic obstructive pulmonary disease (COPD, $n=22$ ). Each point represents data from an individual subject, and some undetectable values are superimposed. Horizontal lines represent the median with the interquartile range. PR3 activity was greater than NE activity in both subject groups. Those with A1AT deficiency had greater PR3 activity than subjects with usual COPD. There was no significant difference in NE activity between the subject groups, although detectable NE activity was only found in six A1AT deficient subjects and one COPD subject. The three A1AT deficient subjects with high NE activity were colonised with Pseudomonas aeruginosa. The greater PR3 activity in sputum from A1AT deficient subjects compared to COPD subjects remained even when subjects colonised with $P$. aeruginosa were excluded $(p=0.006)$.

The median concentration of NE measured by ELISA (free and bound) was less than the median total concentration of its cognate inhibitors both in subjects with A1AT deficiency and usual COPD, as shown in figure 3, and would explain why the majority of sputum samples from both subject groups showed no detectable NE activity. It was not possible to measure the total PR3 concentrations since the ELISA available was only able to detect unbound PR3. Nevertheless, the presence of PR3 activity in the sputum samples indicates that the amount of PR3 exceeds the functional concentration of its inhibitors.

\section{Relationship between PR3 activity and clinical status}

In the 12 subjects with usual COPD who had sputum samples available at the start of an exacerbation and at 8 weeks following the exacerbation when clinically stable, 10 day 1 (exacerbation) samples and six day 56 (stable) samples had detectable PR3 activity and the group values were significantly greater during exacerbation than in the stable state $(p=0.037)$. These data are shown in figure 4 . In this sample of patients, no significant difference was found in NE activity between exacerbation and stable clinical state (data not shown).

Markers of neutrophilic inflammation were measured in the majority $(n=21)$ of usual COPD samples and in a subset of A1AT deficient samples $(n=11)$ for whom sufficient sample remained. The demographics of the COPD subjects in whom the measurements could be made were not significantly different to those for whom they could not. The A1AT 

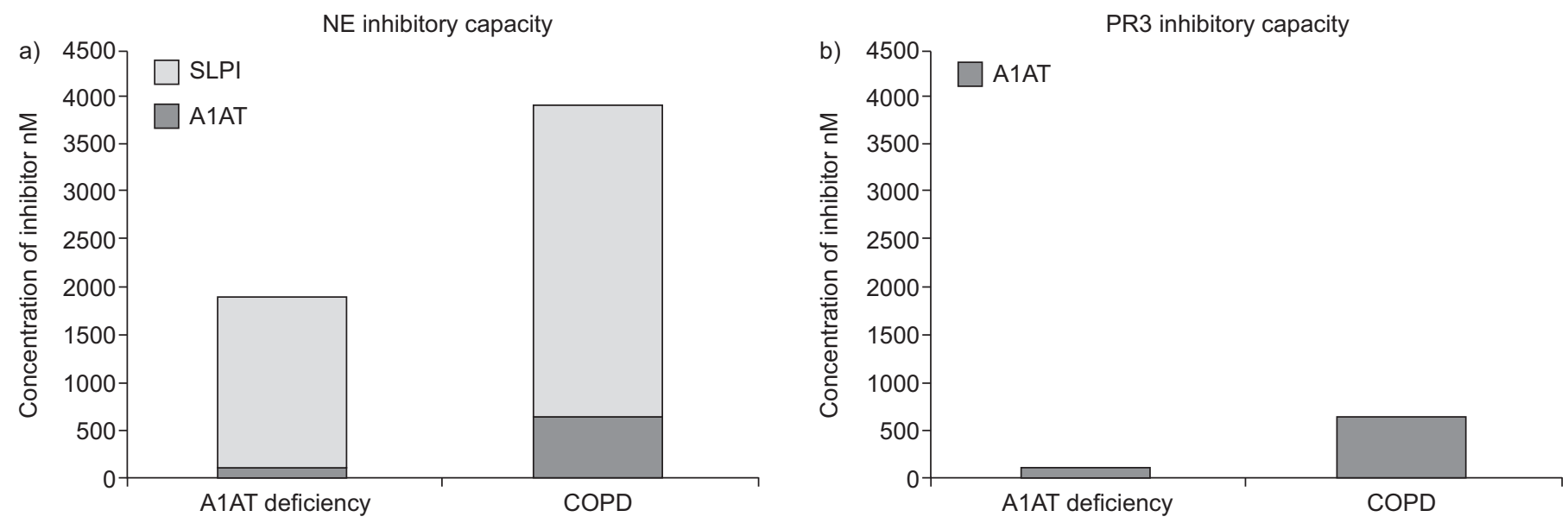

FIGURE 2. Mean concentrations of airway inhibitors in sol phase sputum from subjects with alpha-1-antitrypsin (A1AT) deficiency and usual chronic obstructive pulmonary disease (COPD). The predominant airway inhibitor of neutrophil elastase (NE) in both subject groups was secretory leukoproteinase inhibitor (SLPI). The contribution of elafin was negligible, being found in subnanomolar concentrations in both subject groups, and is therefore not shown on the graphs. Proteinase 3 (PR3) thus has fewer and significantly reduced quantities of its airway inhibitors than NE. The PR3 inhibitory capacity is especially low in A1AT deficient subjects, and indicates why PR3 activity was detected in all samples from this subject group even in the stable clinical state.

deficient subjects who did not have the measurements available were older (59 years versus 50 years: $\mathrm{p}=0.026$ ) and had a lower KCO $(59 \%$ pred versus $82 \%$ pred; $\mathrm{p}=0.031)$ than those whose measurements were available. There were no significant differences in sex, smoking history, smoking status, FEV1 \% pred, FEV1/FVC ratio, residual lung volumes or SGRQ total score.

The concentrations of IL-8 (A1AT deficiency median $6.86 \mathrm{nM}$, IQR 2.02-12.39 nM, COPD median $2.77 \mathrm{nM}$, IQR 1.13-8.24 nM; $\mathrm{p}=0.312$ ), LTB4 (A1AT deficiency median $6.52 \mathrm{nM}$, IQR

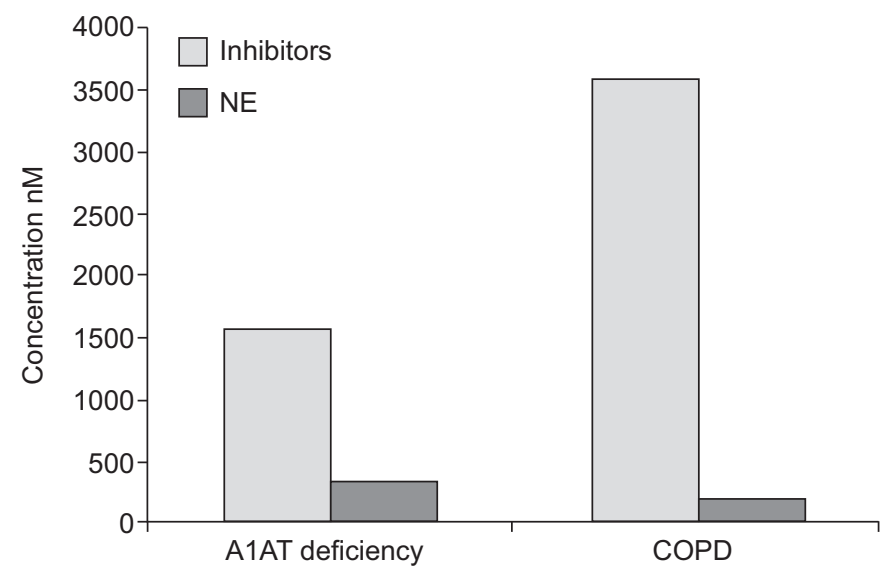

FIGURE 3. Median concentrations of neutrophil elastase (NE; free and bound) and the median sum of its inhibitors alpha-1-antitrypsin (A1AT), secretory leukoproteinase inhibitor and elafin, in sol phase sputum from stable subjects with A1AT deficiency and chronic obstructive pulmonary disease (COPD) are shown. In both subject groups, the total concentration of NE is well below that of its inhibitors, particularly in subjects with usual COPD. The lack of NE activity in the majority of sputum samples is thus probably due to the dominance of its inhibitors. The presence of NE activity in some samples therefore likely reflects significant loss of function of the inhibitors.
4.15-20.02 nM, COPD median $4.72 \mathrm{nM}$, IQR $2.77-17.50 \mathrm{nM}$; $\mathrm{p}=0.463$ ) and the MPO activities (A1AT deficiency median 0.40 units $\cdot \mathrm{mL}^{-1}$, IQR $0.19-0.57$ units $\cdot \mathrm{mL}^{-1}$, COPD median 0.58 units $\cdot \mathrm{mL}^{-1}$, IQR $0.28-1.65$ units $\cdot \mathrm{mL}^{-1} ; \mathrm{p}=0.293$ ) were not significantly different between patient groups.

The correlations between PR3 activity and other parameters are summarised in table 2. PR3 activity correlated positively with NE activity in the A1AT deficient subjects (Spearman's rho $=0.586, \mathrm{p}=0.001$ ) even though NE activity was detectable only in six subjects. PR3 activity correlated positively with IL-8 quantity in both patient groups (A1AT deficiency Spearman's $\mathrm{rho}=0.791, \mathrm{p}=0.004$; COPD Spearman's rho $=0.650, \mathrm{p}=0.001$ ) and MPO activity (A1AT deficiency Spearman's rho $=0.612$,

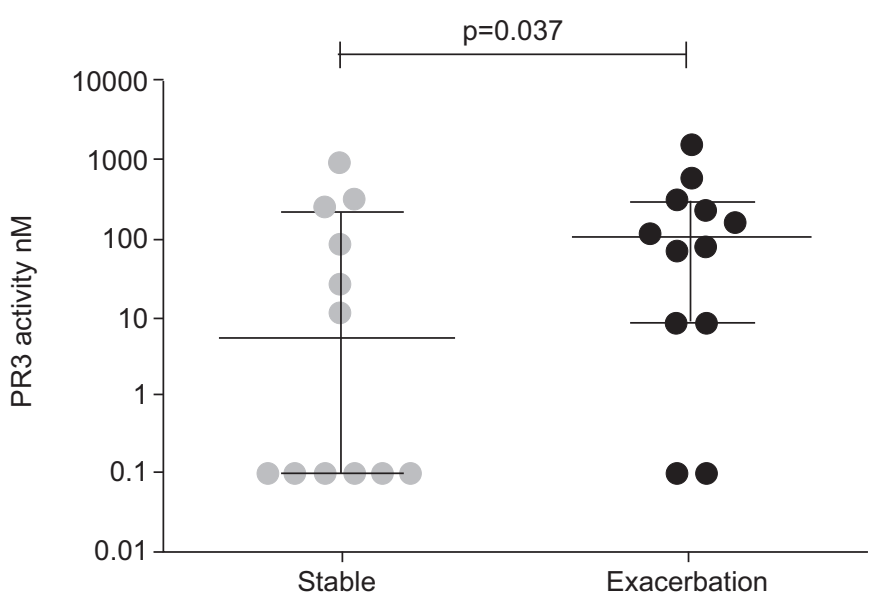

FIGURE 4. Proteinase 3 (PR3) activity in sol phase sputum from 12 subjects with chronic obstructive pulmonary disease taken on day 1 of an exacerbation and day 56 when clinically stable. Horizontal lines represent the median and the interquartile range. PR3 activity was significantly greater during an exacerbation than in the stable clinical state. 
TABLE 2 Correlations of proteinase 3 activity in sol phase sputum

\begin{tabular}{|c|c|c|c|c|}
\hline Diagnosis & Measurement & Samples with measurement $n$ & Spearman's rho & $p$-value \\
\hline \multirow[t]{4}{*}{ A1AT deficiency } & MPO & 11 & 0.612 & $<0.001$ \\
\hline & LTB4 & 11 & 0.291 & 0.385 \\
\hline & SGRQ total & 25 & 0.621 & 0.001 \\
\hline & Total pathogenic bacterial load & 28 (18 positive) & 0.578 & 0.001 \\
\hline \multirow{2}{*}{ COPD } & LTB4 & 21 & 0.434 & 0.049 \\
\hline & Total pathogenic bacterial load & 22 (8 positive) & 0.291 & 0.189 \\
\hline
\end{tabular}

A1AT: alpha-1-antitrypsin; COPD: chronic obstructive pulmonary disease; SGRQ: St George's Respiratory Questionnaire; IL: interleukin; MPO: myeloperoxidase; LTB4: leukotriene B4.

$\mathrm{p}<0.001$; COPD Spearman's rho $=0.799, \mathrm{p}<0.001)$. In the nondeficient COPD group, PR3 activity also correlated positively with LTB4 (Spearman's rho=0.434, $\mathrm{p}=0.049$ ).

PR3 activity was found to correlate with the total pathogenic bacterial load in the A1AT deficient group (Spearman's rho $=0.578, \mathrm{p}=0.001)$. Subjects with $P$. aeruginosa on quantitative microbiological culture had significantly higher NE activity $(p<0.001)$ and PR3 activity $(p=0.025)$ in their sputum compared to subjects who did not. Higher PR3 activity was also found in samples that grew Haemophilus influenzae compared to those that did not $(p=0.003)$ but NE activity was not different. The pathogens isolated in the sputum samples are summarised in table 3 . Some subjects grew more than one pathogen (three A1AT deficient subjects), whilst some did not grow any (10 A1AT deficient subjects and 14 COPD subjects).

A significant correlation was also found between PR3 activity and SGRQ total score in the A1AT deficient subjects (Spearman's rho=0.621, $\mathrm{p}=0.001$ ). The SGRQ scores were not available for the subjects with usual COPD. No correlations were found between PR3 activity and FEV1 (\% pred) or KCO (\% pred) in either subject group.

\section{DISCUSSION}

This study has provided direct evidence that active PR3 is present in sol phase sputum from clinically stable subjects with chronic bronchitis associated with A1AT deficiency as well as nondeficient COPD. PR3 activity was greater than NE activity in these subjects, which likely reflects both the lower airway inhibitory capacity for PR3 and the greater amount of PR3 contained within the azurophilic granules of the neutrophil compared to NE. In addition, the association rate constants of A1AT and elafin for PR3 are orders of magnitude lower than for $\mathrm{NE}$ [30] and, therefore, NE is likely to be preferentially inhibited compared to PR3 in the inflammatory environment. PR3 is also not inhibited by SLPI, which is present in large amounts in respiratory epithelial lining fluid and is the predominant inhibitor of NE in the upper airways. Furthermore, PR3 is capable of degrading SLPI [31], thus potentially enhancing the biological activities of other NSPs that are usually inhibited by SLPI.

PR3 activity was found to be greater during exacerbations than during periods of clinical stability. Exacerbations are often associated with an increased neutrophilic inflammation [32], and this would account for the correlations of PR3 activity with other neutrophilic markers. PR3 activity showed a positive correlation with worsening health status as measured by the SGRQ. PR3 activity is thus a marker of increased airway inflammation and exacerbations, which are both associated with impaired health status in subjects with chronic respiratory disease $[33,34]$ suggesting it is an association rather than cause and effect.

The current study did not, however, demonstrate any relationship between PR3 activity and lung function parameters. In a previous study where PR3 concentration was measured in sputum from 49 subjects with cystic fibrosis, a negative correlation was observed between PR3 concentration and FEV1 (\% pred) [8]. It is possible, therefore, that the present study did not involve sufficient numbers to detect a relationship

\section{TABLE 3 Pathogens isolated in the sputum samples}

\begin{tabular}{lcc} 
Pathogen name & $\begin{array}{c}\text { A1AT deficient } \\
\text { subjects with } \\
\text { positive culture } \mathbf{n}\end{array}$ & $\begin{array}{c}\text { COPD subjects } \\
\text { with positive } \\
\text { culture } \mathbf{~}\end{array}$ \\
\hline Haemophilus influenzae & 9 & 5 \\
Moraxella catarrhalis & 3 & 0 \\
Pseudomonas aeruginosa & 3 & 0 \\
Haemophilus parainfluenzae & 3 & 3 \\
Streptococcus pneumoniae & 1 & 0 \\
Staphylococcus aureus & 2 & 0 \\
Proteus mirabilis & 1 & 0
\end{tabular}

A1AT: alpha-1-antitrypsin; COPD: chronic obstructive pulmonary disease 
between FEV1 and PR3 activity, although other features such as bacterial colonisation (see below) will also complicate this potential relationship.

The current study has directly measured both PR3 and NE activities in biological samples using highly sensitive and specific substrates. Some previously published studies of NSP activities in airway secretions have used non-specific "elastase" substrates (such as N-methoxysuccinyl-Ala-Ala-Pro-Val p-nitroanilide) and potentially measured the combined activities of PR3 and NE [35-37]. Other studies have indirectly measured PR3 activity by measuring the combined activities of PR3 and NE with and without an NE inhibitor [8]. Although SlaaapN has been used to measure NE activity in sputum previously [38], it is less sensitive than the NE specific FRET substrate described here. The direct measurement of individual NSP activities in lung secretions using highly sensitive and specific substrates (as described here) allows the contribution of each NSP to the total proteinase burden to be determined specifically. Although NSPs are released from neutrophils simultaneously upon activation and degranulation, their concentrations can differ due to local factors such as binding to the cell membrane [16], their affinities to endogenous inhibitors at the inflammatory site and their specificities toward peptide or protein substrates [30]. Biological consequences are only likely to occur in the presence (especially the persistent presence) of active enzyme.

The results described here are derived from subjects with A1AT deficiency (PiZZ phenotype) and non-deficient COPD with a chronic bronchitis phenotype. Spontaneous sputum production is associated with greater neutrophilic airway inflammation compared to subjects who do not expectorate, which is not related to smoking status [35]. Sputum production may also be associated with an accelerated decline in FEV1 in COPD [39]. Therefore, although it is important to understand the role of NSPs in this subgroup of patients, the results presented here may be less generalisable to subjects who are not spontaneous sputum producers. Spontaneous sputum collection is non-invasive and minimises dilutional errors found with other techniques, such as bronchoalveolar lavage [40] and induced sputum collection, and may provide critical data in this important phenotype. However, the use of single spontaneously produced sputum samples also has limitations due to the daily variability of sample collection and dilution with nasopharyngeal secretions. This phenomenon has been described in subjects with A1AT deficiency and usual COPD, and could be minimised in future studies by averaging three sequential samples [41]. Nevertheless, despite this potential drawback, the differences and correlations described here remain robust.

In the current study, three subjects with A1AT deficiency were colonised with $P$. aeruginosa and had significantly higher NE and PR3 activities in their sputum compared to subjects who were not. Furthermore, greater PR3 activity was found in samples that grew $H$. influenzae compared to those that did not. Our group has previously shown that in patients with bronchiectasis, $P$. aeruginosa provokes a more intense inflammatory response (with elevated NE and MPO activities) compared to $H$. influenzae, which in turn is greater than that with Moraxella catarrhalis [38]. The six subjects with A1AT deficiency who had detectable NE activity in their sputum had significantly lower concentrations of SLPI and elafin compared to those with undetectable NE activity. Previous work has shown that both SLPI and elafin can be cleaved by NE, particularly in the presence of $P$. aeruginosa $[42,43]$ which may therefore represent an effect rather than a cause. There were no other significant differences observed between the subjects with or without detectable NE activity. Correlations of PR3 activity with airway bacterial load and airway inflammation (NE and MPO activity) support the role of colonisation in airway neutrophilic inflammation (as indicated previously [38, 44]), but highlights the association with PR3 activity, which has the potential to drive the pathophysiological processes that influence COPD and its progression. This enzyme may, therefore, be more important in COPD than has previously been thought.

We have indicated that PR3 activity should be determined when evaluating the proteinase/antiproteinase imbalance in the airways. Whether or not it is central to the process remains to be determined as it remains possible that several proteinases may play a role in the pathogenesis of COPD. In addition to NSPs, both matrix metalloproteinases [45] and cysteine proteinases $[46,47]$ have been associated with tissue destruction in emphysema both directly and indirectly via their interactions with NSPs and their inhibitors. Strategies to reduce the burden of proteinases in the lungs could potentially offer novel therapies for COPD. Recently, JEGOT et al. [48] have developed specific PR3 inhibitors. Selective inhibitors of PR3 may thus provide further insight into its role in disease and (based on the studies presented here) could potentially be of therapeutic value in inflammatory lung diseases.

\section{SUPPORT STATEMENT}

This work was funded using educational grants from Grifols Therapeutics and GlaxoSmithKline. However, the funders had no role in the design of the study, interpretation of the results or production of the manuscript.

\section{STATEMENT OF INTEREST}

Conflict of interest information can be found alongside the online version of this article at www.erj.ersjournals.com

\section{ACKNOWLEDGEMENTS}

The authors wish to thank Peter Nightingale (Queen Elizabeth Hospital, Birmingham, UK) for providing statistical advice.

\section{REFERENCES}

1 Pauwels RA, Rabe KF. Burden and clinical features of chronic obstructive pulmonary disease (COPD). Lancet 2004; 364: 613-620.

2 Abboud RT, Vimalanathan S. Pathogenesis of COPD. Part I. The role of protease-antiprotease imbalance in emphysema. Int J Tuberc Lung Dis 2008; 12: 361-367.

3 Laurell C-B, Eriksson S. The electrophoretic alpha 1 globulin pattern of serum in alpha 1 antitrypsin deficiency. Scand J Clin Lab Invest 1963; 15: 132-140.

4 Rao NV, Wehner NG, Marshall BC, et al. Characterization of proteinase-3 (PR-3), a neutrophil serine proteinase. Structural and functional properties. J Biol Chem 1991; 266: 9540-9548. 
5 Doring G. The role of neutrophil elastase in chronic inflammation. Am J Respir Crit Care Med 1994; 150: S114-117.

6 Lucey EC, Stone PJ, Breuer R, et al. Effect of combined human neutrophil cathepsin $\mathrm{G}$ and elastase on induction of secretory cell metaplasia and emphysema in hamsters, with in vitro observations on elastolysis by these enzymes. Am Rev Respir Dis 1985; 132: 362-366.

7 Kao RC, Wehner NG, Skubitz KM, et al. Proteinase 3. A distinct human polymorphonuclear leukocyte proteinase that produces emphysema in hamsters. J Clin Invest 1988; 82: 1963-1973.

8 Witko-Sarsat V, Halbwachs-Mecarelli L, Schuster A, et al. Proteinase 3 , a potent secretagogue in airways, is present in cystic fibrosis sputum. Am J Respir Cell Mol Biol 1999; 20: 729-736.

9 Sallenave JM. Secretory leukocyte protease inhibitor and elafin/ trappin-2: versatile mucosal antimicrobials and regulators of immunity. Am J Respir Cell Mol Biol 2010; 42: 635-643.

10 Travis J. Structure, function, and control of neutrophil proteinases. Am J Med 1988; 84: 37-42.

11 Ohbayashi H. Neutrophil elastase inhibitors as treatment for COPD. Expert Opin Investig Drugs 2002; 11: 965-980.

12 Korkmaz B, Poutrain P, Hazouard E, et al. Competition between elastase and related proteases from human neutrophil for binding to alpha1-protease inhibitor. Am J Respir Cell Mol Biol 2005; 32: 553-559.

13 Baggiolini M, Bretz U, Dewald B, et al. The polymorphonuclear leukocyte. Agents Actions 1978; 8: 3-10.

14 Bories D, Raynal MC, Solomon DH, et al. Down-regulation of a serine protease, myeloblastin, causes growth arrest and differentiation of promyelocytic leukemia cells. Cell 1989; 59: 959-968.

15 Campanelli D, Detmers PA, Nathan CF, et al. Azurocidin and a homologous serine protease from neutrophils. Differential antimicrobial and proteolytic properties. J Clin Invest 1990; 85: 904-915.

16 Campbell EJ, Campbell MA, Owen CA. Bioactive proteinase 3 on the cell surface of human neutrophils: quantification, catalytic activity, and susceptibility to inhibition. J Immunol 2000; 165 3366-3374.

17 Coeshott C, Ohnemus C, Pilyavskaya A, et al. Converting enzymeindependent release of tumor necrosis factor $\alpha$ and IL-1 $\beta$ from a stimulated human monocytic cell line in the presence of activated neutrophils or purified proteinase 3. Proc Natl Acad Sci USA 1999; 96: 6261-6266.

18 Pendergraft WF 3rd, Rudolph EH, Falk RJ, et al. Proteinase 3 sidesteps caspases and cleaves p21(Waf1/Cip1/Sdi1) to induce endothelial cell apoptosis. Kidney Int 2004; 65: 75-84.

19 Fujinaga M, Chernaia MM, Halenbeck R, et al. The crystal structure of PR3, a neutrophil serine proteinase antigen of Wegener's granulomatosis antibodies. J Mol Biol 1996; 261: 267-278.

20 Korkmaz B, Attucci S, Moreau T, et al. Design and use of highly specific substrates of neutrophil elastase and proteinase 3. Am J Respir Cell Mol Biol 2004; 30: 801-807.

21 Korkmaz B, Attucci S, Juliano MA, et al. Measuring elastase, proteinase 3 and cathepsin $G$ activities at the surface of human neutrophils with fluorescence resonance energy transfer substrates. Nat Protoc 2008; 3: 991-1000.

22 Sapey E, Bayley D, Ahmad A, et al. Inter-relationships between inflammatory markers in patients with stable COPD with bronchitis: intra-patient and inter-patient variability. Thorax 2008 63: 493-499.

23 GOLD. Global Strategy for the Diagnosis, Management and Prevention of COPD, Global Initiative for Chronic Obstructive Lung Disease (GOLD), 2011. Available from: www.goldcopd.org/ guidelines-global-strategy-for-diagnosis-management.html

24 Pye A, Stockley RA, Hill SL. Simple method for quantifying viable bacterial numbers in sputum. J Clin Pathol 1995; 48: 719-724.
25 Nakajima K, Powers JC, Ashe BM, et al. Mapping the extended substrate binding site of cathepsin $G$ and human leukocyte elastase. Studies with peptide substrates related to the alpha 1protease inhibitor reactive site. J Biol Chem 1979; 254: 4027-4032.

26 Korkmaz B, Hajjar E, Kalupov T, et al. Influence of charge distribution at the active site surface on the substrate specificity of human neutrophil protease 3 and elastase. A kinetic and molecular modeling analysis. J Biol Chem 2007; 282: 1989-1997.

27 Wiesner O, Litwiller RD, Hummel AM, et al. Differences between human proteinase 3 and neutrophil elastase and their murine homologues are relevant for murine model experiments. FEBS Lett 2005; 579: 5305-5312.

28 Llewellyn-Jones CG, Harris TA, Stockley RA. Effect of fluticasone propionate on sputum of patients with chronic bronchitis and emphysema. Am J Respir Crit Care Med 1996; 153: 616-621.

29 Kessler E, Israel M, Landshman $\mathrm{N}$, et al. In vitro inhibition of Pseudomonas aeruginosa elastase by metal-chelating peptide derivatives. Infect Immun 1982; 38: 716-723.

30 Ying QL, Simon SR. Elastolysis by proteinase 3 and its inhibition by alpha(1)-proteinase inhibitor: a mechanism for the incomplete inhibition of ongoing elastolysis. Am J Respir Cell Mol Biol 2002; 26: 356-361.

31 Rao NV, Marshall BC, Gray BH, et al. Interaction of secretory leukocyte protease inhibitor with proteinase-3. Am J Respir Cell Mol Biol 1993; 8: 612-616.

32 White AJ, Gompertz S, Stockley RA. Chronic obstructive pulmonary disease. 6: the aetiology of exacerbations of chronic obstructive pulmonary disease. Thorax 2003; 58: 73-80.

33 Brusse-Keizer M, van der Palen J, van der Valk $\mathrm{P}$, et al. Clinical predictors of exacerbation frequency in chronic obstructive pulmonary disease. Clin Respir J 2011; 5: 227-234.

34 Snoeck-Stroband JB, Postma DS, Lapperre TS, et al. Airway inflammation contributes to health status in COPD: a crosssectional study. Respir Res 2006; 7: 140.

35 Gompertz S, Hill AT, Bayley DL, et al. Effect of expectoration on inflammation in induced sputum in alpha-1-antitrypsin deficiency. Respir Med 2006; 100: 1094-1099.

36 Lee RL, Rancourt RC, del Val G, et al. Thioredoxin and dihydrolipoic acid inhibit elastase activity in cystic fibrosis sputum. Am J Physiol Lung Cell Mol Physiol 2005; 289: L875-L882.

37 Simpson JL, Scott RJ, Boyle MJ, et al. Differential proteolytic enzyme activity in eosinophilic and neutrophilic asthma. Am J Respir Crit Care Med 2005; 172: 559-565.

38 Hill AT, Campbell EJ, Hill SL, et al. Association between airway bacterial load and markers of airway inflammation in patients with stable chronic bronchitis. Am J Med 2000; 109: 288-295.

39 Vestbo J, Prescott E, Lange P. Association of chronic mucus hypersecretion with FEV1 decline and chronic obstructive pulmonary disease morbidity. Copenhagen City Heart Study Group. Am I Respir Crit Care Med 1996; 153: 1530-1535.

40 Zedtwitz-Liebenstein K, Schenk P, Apfalter P, et al. Ventilatorassociated pneumonia: increased bacterial counts in bronchoalveolar lavage by using urea as an endogenous marker of dilution. Crit Care Med 2005; 33: 756-759.

41 Stone H, McNab G, Wood AM, et al. Variability of sputum inflammatory mediators in COPD and alpha-1-antitrypsin deficiency. Eur Respir J 2012; 40: 561-569.

42 Weldon S, McNally P, McElvaney NG, et al. Decreased levels of secretory leucoprotease inhibitor in the Pseudomonas-infected cystic fibrosis lung are due to neutrophil elastase degradation. J Immunol 2009; 183: 8148-8156.

43 Guyot N, Butler MW, McNally P, et al. Elafin, an elastase-specific inhibitor, is cleaved by its cognate enzyme neutrophil elastase in sputum from individuals with cystic fibrosis. J Biol Chem 2008; 283: 32377-32385. 
44 Marin A, Garcia-Aymerich J, Sauleda J, et al. Effect of bronchial colonisation on airway and systemic inflammation in stable COPD. COPD 2012; 9: 121-130.

45 Churg A, Zhou S, Wright JL. Matrix metalloproteinases in COPD. Eur Respir J 2012; 39: 197-209.

46 Mason RW, Johnson DA, Barrett AJ, et al. Elastinolytic activity of human cathepsin L. Biochem J 1986; 233: 925-927.
47 Lesser M, Padilla ML, Cardozo C. Induction of emphysema in hamsters by intratracheal instillation of cathepsin B. Am Rev Respir Dis 1992; 145: 661-668.

48 Jegot $G$, Derache C, Castella $S$, et al. A substrate-based approach to convert SerpinB1 into a specific inhibitor of proteinase 3, the Wegener's granulomatosis autoantigen. FASEB J 2011; 25: 30193031. 